\title{
Frank Ziegler
}

\section{„... und jezt gerne einen Ruhigen Platz annehmen würde“ Edmund von Weber und seine Familie zwischen Rheinland, Lippe und Franken (1825-1831)}

Carl Maria von Webers Halbbruder Edmund hatte seit 1820 an verschiedenen Theatern als Musikdirektor gearbeitet: in Lübeck (Wintersaison 1820/21), Danzig (Oktober 1821 bis März 1824, ${ }^{1}$ mit Abstechern nach Elbing), Königsberg (bis zum Sommer 1824) ${ }^{2}$ und schließlich bis Spätsommer 1826 unter Direktor Friedrich Sebald Ringelhardt, dem Ehemann von Edmunds Nichte Victorine Ringelhardt, geb. Weyrauch, in Köln und Aachen. ${ }^{3} 1826$ versuchte er, für sich sowie seine Töchter Therese (1809-1884) und Josephine (geb. 28. Oktober 1810) eine neue Wirkungsstätte zu finden. Mehrere Bewerbungsbriefe Edmund von Webers aus dieser Zeit sind bekannt: Beim Kasseler Hofkapellmeister Louis Spohr fragte er an, ob für ihn und seine „beiden talentvollen Töchter keine Stellung an der Casseler Hofbühne wäre“. ${ }^{4}$ Am 5. Juni 1826 bot er von Aachen aus einem unbekannten Empfänger an, er würde bei einem Engagement seiner älteren Tochter Therese als Sängerin selbst „eine Stelle als Contra-Bass im Orchester annehmen“, denn „Musikdirektor bei einer reisenden [...] Gesellschaft zu sein, ist auch

1 Der Wunsch Edmund von Webers, anschließend als Nachfolger des 1823 pensionierten Johann Peter C. Wiele Kapellmeister in Neustrelitz zu werden, ging trotz der Empfehlung durch Carl Maria von Weber (vgl. dessen Brief an Carl Graf Brühl vom 13. Februar 1824, D-B, 55 Ep 302) nicht in Erfüllung.

2 Auf der Reise von Königsberg nach Köln besuchte er Hamburg, wo sein ältester Bruder Fridolin von Weber lebte; vgl. Jürgen Neubacher, Die Webers, Haydn und Der Aepfeldieb. Eine Untersuchung der Musikhandschrift ND VII 168 der Staats- und Universitätsbibliothek Hamburg Carl von Ossietzky, in: Festschrift Christoph-Hellmut Mahling zum 65. Geburtstag, hg. von Axel Beer, Kristina Pfarr und Wolfgang Ruf, Tutzing 1997, Bd. 2, S. 989-1008, hier S. 1006 (Anm. 65).

3 Eine finanzielle Starthilfe Carl Maria von Webers für seinen Bruder in Köln ist durch Webers Tagebuch dokumentiert, wo am 24. Dezember 1824 festgehalten wurde: „Euryanthe an Ringelhart abgesendet. und Honorar dem Bruder Edmund geschenkt". Die Kölner Erstaufführung unter Ringelhardts Direktion fand allerdings erst am 19. Oktober 1829 statt; vgl. Otto Heyden, Das Kölner Theaterwesen im 19. Fahrhundert. 1814-1872, Emsdetten 1939, S. 95. In Aachen wurde das Werk unter Ringelhardt sogar erst 1831 gegeben; vgl. Bernhard Poll, Zeittafel zur Aachener Musikgeschichte im 19. und 20. Jahrhundert, in: Beiträge zur Musikgeschichte der Stadt Aachen, Köln u. a. 1954 (Beiträge zur rheinischen Musikgeschichte 6), S. 4868, hier S. 49.

4 Leo Liepmannssohn, Katalog zur Auktion am 15./16. Oktober 1894 (Nachlass Louis Spohr), Nr. 603 (S. 72 f.), ohne genaue Datumsangabe (Aachen 1826); Verbleib des Originals unbekannt. 
kein grosses Glück“. ${ }^{5}$ Schließlich ging am 5. Oktober 1826 ein Schreiben aus Köln an Friedrich Ludwig Schmidt in Hamburg, in dem Edmund von Weber sich als Dirigent oder Kontrabassist, seine Frau Therese, geb. Mack, als Schauspielerin („spielt das Fach der komischen Mütter in Stück und Oper ist auch etwas musikalisch“) und seine ältere Tochter als Sängerin und Schauspielerin („sehr wohl gebauth, in mereren Opera und Stücken einstudirt und sehr musikalisch") anpries. ${ }^{6}$ Zunächst blieb die Suche erfolglos, und so plante der stellungslose Musiker, wie sein Kollege Albert Lortzing berichtete, vorerst „auf seine eigene Faust [zu] reisen“, um „wahrscheinlich Conzerte auf dem Kontrabaß [zu] geben". 7 Erst 1827 fand sich für Therese von Weber eine Anstellung als Sängerin am Detmolder Hoftheater unter Direktor August Pichler, und so zog die Familie in die lippische Residenzstadt, auch wenn der nunmehr sechzigjährige Edmund von Weber dort zunächst genauso wenig eine feste Anstellung erhielt, ${ }^{8}$ wie seine Frau Therese und seine jüngste Tochter.

Die ältere Tochter Therese hatte bereits bei der Ringelhardt'schen Truppe erste Erfahrungen als Solistin gesammelt: In Aachen hatte sie am 1.Januar 1826 (noch 16-jährig) das Lieschen in Paisiellos Schöner Müllerin gespielt und „sich als Anfängerin lobenswerthe Mühe" gegeben;" es folgten u. a. Auftritte als Knabe Elamir in Salieris Axur (21. Januar) ${ }^{10}$ und als Zerline in Mozarts Don Giovanni (8. Juni). ${ }^{11}$ Besonders gegen Ende der Aachener Sommerspielzeit 1826 war sie offenbar aufgrund personeller Engpässe eine unverzichtbare Stütze, hatte doch Albert Lortzing seinem Freund Schäfer berichtet: „wir brauchen sie unter uns gesagt, wie das liebe Brod.“ ${ }^{12}$ So konnte Therese sich auch als Cherubino in Figaros Hochzeit und Ännchen im Freischütz „nicht ohne einigen Erfolg" versuchen. ${ }^{13}$ Der Korrespondent des Rheinisch-Westphälischen Anzeigers urteilte wohlwollend:

5 Mehrfach bei Leo Liepmannssohn, u. a. Katalog 163 (ca. 1906/07), Nr. 873 (S. 79); Katalog 174 (ca. 1909/10), Nr. 2218 (S. 126); Verbleib des Originals unbekannt.

6 Vgl. Neubacher, Die Webers, Haydn und Der Aepfeldieb (wie Anm. 2), S. 1005 f. (Anm. 65, Zitate auf S. 1006) und S. 996 (Anm. 30, dort Signatur).

7 Brief vom 21. August 1826 an Ludwig Schäfer; vgl. Albert Lortzing, Sämtliche Briefe. Historisch-kritische Ausgabe, hg. von Irmlind Capelle, Kassel 1995 (Detmold-Paderborner Beiträge zur Musikwissenschaft 4), S. 5.

8 Vgl. Joachim Veit, Art. (Franz) Edmund (Kaspar fohann Nepomuk foseph Maria) Weber, in: The New Grove. Dictionary of Music and Musicians, 2. edition, hg. von Stanley Sadie, Bd. 27, London 2001, S. 134.

9 Rheinische Flora, Blätter für Kunst, Leben, Wissen und Verkehr, Aachen, Jg. 2, Beilage zu Nr. 2 (3. Januar 1826); alle Beilagen ohne Paginierung.

10 Ebd., Jg. 2, Beilage zu Nr. 14 (24. Januar 1826): ihr „Gebet wurde mit verdientem Beifall belohnt“.

11 Ebd., Jg. 2, Beilage zu Nr. 91 (11. Juni 1826): „Das Viertelstimmchen [...] verfloß in dem großen Hause, wie ein Bach im Meere."

12 Lortzing, Briefe (wie Anm. 7), S. 5.

13 Vgl. Historische Übersicht des Theaters in Aachen von 1825 bis Ende 1828, in: Almanach für's Aachener Stadt-Theater auf das Fahr 1829, hg. von Dr. Arendt, Aachen 1829, S. $74 \mathrm{f}$. 
Dem. v o n W e b e r, die Tochter unseres Musikdirektors, ist noch Anfängerin in der Oper, ein junges liebenswürdiges Mädchen, welche durch ihr Auftreten als Emilie im Othello, Cherubin im Figaro, und Zerline im Don Juan gezeigt hat, daß mit der Zeit eine tüchtige Sängerin aus ihr werden kann. ${ }^{14}$

Nach dem Abgang ihres Vaters Edmund blieb sie im Herbst 1826 vorerst bei der Ringelhardt'schen Truppe in Köln und entwickelte sowohl im Schauspiel als auch in der Oper zunehmend Routine. ${ }^{15}$

Unter August Pichler debütierte Therese von Weber Anfang 1827 in Münster; ${ }^{16}$ ihre erste Wochengage von 12 Talern erhielt sie am 29. Januar für die fünfte Kalenderwoche (29. Januar bis 4. Februar 1827) ausgezahlt. ${ }^{17}$ Zudem wurde ihr einmalig ein Reisegeld („Für Fracht, der, Freulein von Weber angehörigen Sachen, von Cöln nach Münster“) von mehr als 8 Talern gezahlt. ${ }^{18}$ Ein dortiger Korrespondent urteilte, dass „der Gesang dieser jungen Künstlerinn in einer recht guten Schule gebildet worden, ihre Intonation rein und richtig, dagegen ihre Stimme noch schwach, jedoch rein und klangvoll sei.“ Weiter heißt es:

Daß Fräulein von Weber in ihrem Spiele noch nicht den Gipfel der Kunst erstiegen habe, vielmehr noch Anfängerin, sowohl in dieser Beziehung als in der Kunstfertigkeit des Gesanges sei, darüber ist der kunst- und sachverständige Theil des hiesigen Publikums sich völlig einig; allein nur durch zweckmäßige Aufmunterung kann das aufkeimende Talent zum weiteren Fortschreiten auf der angefangenen Kunstbahn ermuthiget werden. ${ }^{19}$

14 Korrespondenzbericht aus Aachen von Anfang August, gez. „Spektator d. J., in Bd. 46, Beilage zu Nr. 69 (30. August 1826), Sp. 1528.

15 Vgl. Rheinische Flora (wie Anm. 9), Jg. 2, Nr. 161 (14. Oktober 1826), S. 644: Mitwirkung in Schreyvogels Einrichtung der Donna Diana am 6. Oktober; Beilage zu Nr. 164 (19. Oktober 1826): Zerline im Don Giovanni am 11. Oktober („diese angenehme Erscheinung belebt sich allmählig, wie Pygmalion’s Stein, aber leider sehr allmählig“); Beilage zu Nr. 175 (9. November 1826): Mitwirkung in Kotzebues Erbschaft am 5. November; Beilage zu Nr. 181 (19. November 1826): Emilia in Rossinis Otello am 15. November („ein günstiges Zeugniß ihres Fortschreitens").

16 Das Detmolder Hoftheaterensemble spielte 1827 von Januar bis April in Münster und ab Mitte April in Osnabrück; vgl. Lortzing, Briefe (wie Anm. 7), S. 10.

17 Lippische Landesbibliothek Detmold (D-DT), Theaterakten, Signatur TA 101, 1827: Gagenbuch Nr. 23 Therese von Weber pro 1827. Alle Angaben zu diesem Aktenbestand verdanke ich Irmlind Capelle, die gemeinsam mit Kristina Richts im Projekt „Detmolder Hoftheater 1825-1875“ u. a. die dortigen archivalischen Quellen erschließt. Die Angaben entsprechen dem derzeitigen Bearbeitungsstand; das Auftauchen weiterer Quellen im Rahmen der Arbeit ist nicht auszuschließen.

18 Ebd., TA 1, diverse Ausgaben 1827, S. 81 (Nr. 33).

19 Rheinische Flora (wie Anm. 9), Jg. 3, Nr. 20 (16. Februar 1827), S. 80. Derselbe Bericht erschien offenbar nochmals an anderer Stelle; die hier zitierte Passage griff der Autor einer ungezeichneten „Entgegnung“ 
Obgleich der Münsteraner Berichterstatter betonte, dass die Direktion der jungen Künstlerin „ohne Zweifel [...] nur [...] jugendliche, sogenannte zweite Gesangparthieen "übertragen werde, wurde sie nachfolgend in Detmold auch in größeren Rollen besetzt. Der gestrenge Kritiker Christian Dietrich Grabbe beurteilte sie als Anna in Boieldieus Oper Die weiße Frau in der Aufführung am 16. September 1827 daher wenig freundlich: „ihr Spiel ist matt, ihre Stimme dünn, noch von wenig Schule zeugend [...]. Als e r s t e Sängerin genügt sie nicht,,$^{20}$-aber daß sie Ermunterung verdient, daß bei ihrer Jugend sich das Bessere entwickeln kann, gesteht Ref. gern ein. ${ }^{121}$ Auch als Amenaide in Rossinis Tancred drei Tage später zeigte sie laut Grabbe „keine Bravour".22 An Gubitz' Gesellschafter berichtete Grabbe Anfang 1828 immerhin, dass das Detmolder Ensemble „ein trefliches, angehendes Talent, von Schule und Bildung, in Fräulein v. Weber" besitze, ${ }^{23}$ und an die Dresdner Abend-Zeitung meldete er im April 1828:

Fräulein v. W e b e r [...] wird in zweiten Parthieen jede Bühne zieren, und man gesteht dieß um so herzlicher, als sie durch fleißiges Streben sich Tag für Tag die Gunst des Publikums mehr gewinnt. Sie aber als erste Sängerin zu benutzen, wie es denn oft geschieht, heißt sie aus ihrer Sphäre reißen, sie in Gesang und Spiel für dieselbe verderben, und dagegen das Publikum mit Bravour-Parthieen ermüden, denen nichts fehlt als die Bravour. Mit einer so dünnen Stimme als die des Fräul. v. Weber die Recitative und Arien der Donna Anna vortragen zu hören, erregt die Empfindung, als wenn statt des Stromes ein Bächlein dahinrauschte. Weit lieber hätten wir die Sängerin als Fanchon gesehen, besonders da sie auch nicht übel spielt [...]. ${ }^{24}$

aus Münster vom 6. Februar 1827 auf, die im Rheinisch-Westphälischen Anzeiger, Bd. 47, Beilage zu Nr. 13 (14. Februar 1827), Sp. 247-250 erschien. Dort (Sp. 248) ist zur Quelle der Aussage angegeben: „Ueber ihren Werth hat sich der Verfasser des im Westphälischen Merkur sub No. 8 enthaltenen Aufsatzes wahr und richtig ausgesprochen, und wir müssen dieser Ansicht in sofern unbedingt beitreten."

Das wiederholte Betonen der Berichterstatter, dass Therese von Weber keinesfalls für erste Partien geeignet sei, könnte auch Reaktion auf eine Falschmeldung des Kölner Rheinischen Unterhaltungsblatts (Jg. 3, 1827, Nr. 12) sein. Dieser widersprach der anonyme Münsteraner Bericht vom 6. Februar im Rheinisch-Westphälischen Anzeiger (vgl. Anm. 19, Sp. 247 f.), nach welchem die Debütantin nicht, wie im Unterhaltungsblatt behauptet, ,als Agathe im Freischützen rauschend herausgerufen, in den Dorfsängerinnen als Rosine aufgetreten“ und „am allerwenigsten als e r s t e Sängerin engagirt" sei. Christian Dietrich Grabbe, Werke, Bd.4, Emsdetten 1966, S. 69 (Bericht aus der Westphalia vom 29. September 1827), siehe auch http://www.grabbe-portal.de [Stand: 30. Nov. 2015].

Ebd., S. 72 .

Ebd., S. 77.

24 Ebd., S. 80 f. Der mit „B. B“. gezeichnete Originalbeitrag erschien in der Abend-Zeitung 1828, Nr. 99 (24. April), S. 396, Nr. 100 (25. April), S. 400, Nr. 101 (26. April), S. 404 (dort das obige Zitat) und Nr. 102 (28. April), S. 408 sowie nochmals in: Westphalia, eine Zeitschrift für unbefangene Leser aus allen Ständen, 
Diese Fehlbesetzungen dürften dem Ehrgeiz der Familie von Weber zuzuschreiben sein. Von großen Ambitionen zeugt auch die Stückwahl zur Benefiz-Vorstellung für Therese von Weber am 14. März 1828 in Münster: Mozarts Entführung aus dem Serail. ${ }^{25}$ Albert Lortzing schrieb seinen Eltern am 2. Juni 1828 aus Osnabrück:

Mit unsrer Weber will es nicht beßer werden. Sie ist vergangene Woche nach 4 Wöchentlicher Pause wieder aufgetreten als Rosine im Barbier v. Sevilla, aber sie kann das $(G)$ oben nicht ohne Anstrengung nehmen, und aushalten gar nicht, sie singt jetzt beinahe nur alle 14 Tage einmal, weil wir noch andre Sängerinnen haben [...]. Wenn's mit der Weber nicht beßer wird, so müßen sie grade zu betteln gehn. - Jetzt schimpft die Bagage, ihre Tochter habe zu viel singen müßen, sehr wahr! Aber hat man einer Andern eine erste Parthie gegeben, so haben sie Scandal gemacht und geschrien, ihre Tochter würde zurückgesetzt. ${ }^{26}$

Nachfolgend gelang offenbar der Fachwechsel und damit auch die stimmliche Konsolidierung, wie wiederum Lortzing seinen Eltern am 8. November 1828 aus Münster mitteilte: „Die Weber benimmt sich sehr klug. Sie macht auf die Bravour=parthien keine Ansprüche mehr, sondern will sich mehr in das Spielparthienfach, wo leichter Gesang vorherrschend ist, hineinwerfen, was sie auch erhalten wird [...] ${ }^{\text {“. }}{ }^{27}$ Tatsächlich gab Therese von Weber kurz darauf in der Einstudierung des Oberon eine vergleichsweise kleine Partie: den Puck..$^{28}$

hg. von Dr. F. P. Glanzow, Herford, Jg. 7, Nr. 43 (25. Oktober 1828), S. 342 f., Nr. 44 (1. November 1828), S. 350 f. und Nr. 45 (8. November 1828), S. 358-360 (obiges Zitat auf S. 358 f.).

25 Vgl. Westfälischer Merkur, Münster, 1828, Nr. 32 (13. März). Während der Münsteraner Spielzeiten der Detmolder Gesellschaft vom 4. Januar bis 1. April, von Anfang September bis 25. November sowie im Dezember 1828 standen in den dortigen Konzerten erstaunlich viele Kompositionen von Carl Maria von Weber auf dem Programm. Das lässt zumindest die Vermutung zu, dass Edmund von Weber in die Planungen eingebunden war bzw. Aufführungs-Material aus seinem Besitz zur Verfügung stellte. Georg Schmidt annoncierte für den 11. März ein Finale aus der Euryanthe, für den 15. März und den 11. Oktober die Oberon-Ouvertüre, für den 18. Dezember eine Sopran-Arie aus Oberon sowie für den 27. Dezember nochmals die Oberon-Ouvertüre und ein Finale aus Euryanthe; vgl. ebd., Beilage zu Nr. 23 (21. Februar), Beilage zu Nr. 31 (11. März), Nr. 146 (5. Oktober), Nr. 187 (16. Dezember) und Nr. 192 (25. Dezember). Außerdem spielte der Münsteraner Hornist Rammelsberg am 30. Dezember Webers Horn-Concertino (im selben Konzert erklang die Freischütz-Ouvertüre); vgl. ebd. Nr. 194 (28. Dezember).

26 Lortzing, Briefe (wie Anm. 7), S. 14.

27 Ebd., S. 19.

28 Vgl. das Kostümbuch in D-DT, Theaterakten, TA 54, S. 273; siehe hierzu den Beitrag von Irmlind Capelle im vorliegenden Band. Die Erstaufführung des Oberon in Münster fand am 19. November 1828 statt; vgl. Westfälischer Merkur, Münster, 1828, Nr. 170 (16. November). Am 14. Dezember 1828 folgte die Detmolder Erstaufführung. 
Die ältere Tochter war also in Detmold versorgt; mit ihrer Jahreseinnahme von 600 Talern ${ }^{29}$ lag sie, verglichen mit ihren Kollegen, im „Mittelfeld“, einer Anfängerin mit herausgehobenen Partien angemessen. Daneben stand ihr offenbar jährlich ein Benefiz zu: Schon Anfang 1827, unmittelbar nach ihrer Ankunft, erhielt sie die komplette Einnahme (abzüglich Unkosten) einer Vorstellung von Peter von Winters Oper Das unterbrochene Opferfest (53 Taler, 14 Groschen), ${ }^{30} \mathrm{im}$ Jahr darauf die halbe Einnahme der bereits genannten Vorstellung der Mozart'schen Entführung am 14. März 1828 (immerhin 57 Taler, 23 Groschen). ${ }^{31}$ Außerdem gab es mehrfach Zusatzzahlungen, so im Juni 18275 Taler, 16 Groschen Extra-Vergütung „für eine Rolle in der schönen Müllerin“"von Paisiello, ${ }^{32}$ in Einzelfällen Garderobengeld ${ }^{33}$ sowie 1828 eine Gratifikation von 24 Talern „Auf höchsten Befehl“. ${ }^{34}$

Vater Edmund von Weber suchte hingegen weiterhin eine neue Anstellung, wie ein im Archiv des Verlages Schott erhaltener Brief bezeugt. ${ }^{35}$ In der von Gottfried Weber herausgegebenen Musikzeitschrift Caecilia hatte der stellungslose Musiker folgende „Anzeige“ gefunden, die ihm offenbar vielversprechend erschien:

Ein Musiklehrer wird gesucht.

In einer der angenehmsten Städte des Grossherzogthums Nieder-Rhein, würde ein Musiklehrer, welcher gründlichen Unterricht auf dem Clavier und im Gesange ertheilen könnte, besonders wenn er noch Violin oder Bass spielte, ein reichliches Auskommen finden. Nähere Auskunft ertheilt die Musikhandlung von B. Schotts Söhnen, wenn man sich portofrei an sie wendet. $^{36}$

Darauf bezugnehmend schrieb Edmund von Weber am 10. November 1827 (in recht fehlerhaftem Deutsch) an den Mainzer Verlag, in dem auch die Caecilia erschien:

$29 \quad D-D T$, Theaterakten, TA 1 (Ausgaben-Journal 1826 und 1827, Gagen), S. 31, TA 3 (Haupt-Ausgabe zur Theater-Kasse), S. 4 (1828) und S. 93 (1829). Die anteiligen Einnahmen 1827 (ab Ende Januar, vgl. Anm. 17) und 1829 (bis Ende August) betrugen 580 bzw. 450 Taler.

Ebd., TA 83, 1837: Beläge zur Theater-Rechnung, Tit. V Für Benefize und Gastdarstellungen, Nr. 3 (Abrechnung vom 28. Januar 1827).

31 Ebd., TA 3 Haupt-Ausgabe zur Theater-Kasse 1828 und 1829: Benefize 1828, Nr. 8; die Gesamteinnahme der Vorstellung betrug 137 Taler, 4 Groschen, vgl. TA 28, Einnahme-Journal 1828-1835, S. 5 (unter 14. März).

32 Ebd., TA 1, Ausgaben-Journal 1826 und 1827, Tit. IV diverse Ausgabe 1827, S. 92 (Nr. 214).

33 Ebd., TA 1, Ausgaben-Journal 1826 und 1827, Tit. II Ausgabe für Bibliothek und Garderobe 1827, Nr. 74: 1 Taler, 10 Groschen für die Anfertigung von einem Paar Schuhe; TA 1, Ausgaben-Journal 1826 und 1827, Tit. IV diverse Ausgabe 1827, S. 100 (Nr. 292): 25 Taler „Vergütung für Garderobe“.

34 Ebd., TA 3 Haupt-Ausgabe zur Theater-Kasse 1828 und 1829, Tit. IV diverse Ausgabe 1828, S. 69 (Nr. 450).

35 D-B, Schott-Archiv, Nr. 26000; auf der Rückseite seitenverkehrt der Verlagsvermerk: „von Weber | Lippe Detmold | 10 Nov. $1827^{\prime \prime}$.

36 Intelligenzblatt zur Caecilia 1827 [eigentlich zu Bd. 7 = Heft 25 bis 28, Mainz 1828], Nr. 25, S. 33. 
An die Musikhandlung v. B: Schotts Söhnen

Da man einen Musiklehrer in Großherzogthums des Nieder Rhein wünscht zu haben, so bin [ich] so frey um der nähren Ausku[n]ft zu bitten. ich bin der Bruder [von] Carl Maria v: Weber, der 20 Jahre als MusikDirektor bey den Besten Theaters gestanden hat, ${ }^{37}$ und jezt gerne einen Ruhigen Platz annehmen würde. ich bin zwar verheirathet da aber meine Tochter eine Brave Sängerin ist und hier beym Hoftheater angagiert ist, so ist Sie mit Mutter und Schwester versorgt. Die verlangten eigenschaften des Musiklehre[r]s besitze alle, ich gebe auf allen seiten Instrumenten Unterricht, so wie in Gesang wo ich schon viele Sängerinnen gebildet habe, ich kann mich auch auf das Zeugniß des $\mathrm{H}$ : Capellmeisters Haßloch wie des Capellmeisters Detousch, die beyde in Ihrer nähe sind, berufen.

\author{
ich habe die Ehre mich zu \\ nennen Edmund von Weber \\ Musikdirektor
}

\title{
Lippe Detmold.
}

d $10^{\text {ten }}$ Nov: 1827 .

Vor einen Jahre hatte das Vergnügen Sie selbst in Mainz zu sprechen, ich kaufte noch die 4händige Ouvertur aus Oberon $^{38}$

[verso:] N: S:

Ich bitte um baldige Antwort, die Türkischen Instrumente die hieher kommen habe ich vorgeschlagen, und ich hoffe das ich Ehre damit einlegen werde, die Pauken die wir damals nach Aachen bekamen, waren recht gut.

\section{von Weber \\ beim Fürstlichen Hofftheater | in | Lippe Detmold.}

37 Als Musikdirektor war Edmund von Weber bereits 1810/11 am Bergischen Theater in Elberfeld und Düsseldorf sowie 1811/12 unter Prinzipal Georg Dengler in Freiburg/Breisgau und Bern tätig, während seiner nachfolgenden Jahre in Bern (bis 1819) sind jedoch keine Theater-Kontakte bezeugt; vgl. Ryuichi Higuchi und Frank Ziegler, „Fürchte Gott! Und wandle den Weg der Tugendt“. Das Stammbuch Edmund von Webers als biographische Quelle, in: Weberiana. Mitteilungen der Internationalen Carl-Maria-vonWeber-Gesellschaft e. V. 18 (2008), S. 5-32, hier S. 28 f., 32.

38 Vermutlich ist das bei Schlesinger in Berlin erschienene vierhändige Arrangement der Ouvertüre von Johann Philipp Schmidt (VN: 1382), erschienen im August 1826, gemeint. Auf einem Lieferschein vom 22. September 1826 ( $D$ - $B$, Schott-Archiv, in Nr. 2557) ist die Übersendung von sechs Exemplaren der Ausgabe von Schlesinger an Schott vermerkt. 
mit H. Capelmeister Destousch war ich als Schüler bey Haydn, ${ }^{39}$ und den H: Capellmeister Haßloch habe erst vor einen Jahre in Darmstadt besucht ${ }^{40}$ wenn es nöthig wäre so könte sehr Bald die Stelle annehmen.

Dass Edmund von Weber die gewünschte Anstellung nicht erhielt, verwundert nach diesem recht konfusen Brief mit einem Nachtrag zum Haupttext, einem Nachsatz und einem Nachtrag zum Nachsatz kaum. Zudem passte sein Bewerbungsprofil (Lehrer für alle Streichinstrumente und Gesang) nicht genau zu den Ausschreibungsbedingungen (in erster Linie Lehrer für Klavier und Gesang, evtl. mit zusätzlicher Befähigung für Geige oder Bass). Und sicher war es alles andere als geschickt, gleich im ersten Satz zu erwähnen, in erster Linie an einem ruhigen Posten zur Alterssicherung interessiert zu sein, anstatt die jahrelangen Erfahrungen als Lehrer (u. a. in Bamberg 1809, Bern ab 1812 und Lübeck 1819/20) zu betonen. Vielleicht rechnete Weber aber auch damit, dass sich seine in den letzten Jahren zunehmende Amtsmüdigkeit ohnehin herumgesprochen hatte.

Während seiner Anstellung bei Ringelhardt war Edmund von Weber sowohl in Köln als auch in Aachen immer wieder das Ziel harscher öffentlicher Angriffe von seiten der ortsansässigen Kritiker gewesen. Zu seinem Kölner Benefiz am 3. Februar 1825 (C. M. von Webers Freischütz) liest man noch: „Hr. v. Weber wußte die Achtung des zahlreich versammelten Publikums durch ein gut besetztes Orchester in einem ausgezeichneten Grade zu würdigen“. 41

Doch zur Aufführung von Hérolds Wunderglöckchen am 21. Februar schrieb derselbe Kritiker: „Das Orchester schoß mehrere Böcke. Der Musikdirektor spielte mit seinem Fidelbogen auf dem Notenbuche ein die Hauptsache störendes Nebenkonzert.“ 42

39 Edmund von Weber war von Anfang 1787 bis Mai 1788 Schüler Joseph Haydns in Esterháza; vgl. Higuchi/ Ziegler, Das Stammbuch Edmund von Webers (wie Anm. 37), S. 10, 29. Franz Seraph von Destouches (17721844), ab 1787 für vier Jahre Kompositions-Schüler Haydns und Cellist der Hofkapelle in Esterháza, war von 1820 bis zu seiner Pensionierung 1841 Hofkapellmeister in Homburg.

Gemeinsam mit Theodor Haßloch (1769-1829) war Edmund von Weber 1797 bis 1799 am Kasseler Hoftheater engagiert; vgl. Higuchi/Ziegler, Das Stammbuch Edmund von Webers (wie Anm. 37), S. 19, 31. Haßloch war seit 1810 in Darmstadt als Regisseur, Tenor und Schauspieler engagiert, seit Oktober 1811 als Hofmusikmeister; vgl. Dismas Fuchs, Chronologisches Tagebuch des Großherzoglich Hessischen Hoftheaters, von der Begründung bis zur Auflösung desselben. Ein Beitrag zur Geschichte der deutschen Schaubühnen, Darmstadt 1832, S. 14, 27 f., 43.1813 wurde er Hofkapellmeister. E. von Webers Aufenthalt 1826 in Darmstadt ist ansonsten nicht dokumentiert, passt aber zu Lortzings Hinweis auf dessen Reiseplanungen im Brief vom 21. August 1826 (vgl. Anm. 7) in Zusammenhang mit der Engagementssuche.

41 Rheinische Flora (wie Anm. 9), Jg. 1, Nr. 29 (19. Februar 1825), S. 116. Der Kölner Kritiker zeichnete seine Beiträge mit „Attento“ (Aufgepasst).

42 Ebd., Jg. 1, Beilage zu Nr. 34 (27. Februar 1825); die Beilagen dieser Zeitschrift sind durchgehend ohne Paginierung. In derselben Ausgabe heißt es zu C. M. v. Webers Preciosa am 20. Februar: „Das Orchester spielte unter der Direktion des H. E d m u n d v. W e b e r mit vieler Anstrengung“. 
Ähnliche Urteile finden sich mehrfach: Bei Rossinis Tancred am 17. Januar wurde bemängelt, dass die Chöre „so miserabel einstudirt [waren], daß mehrere Störungen nicht unbemerkt blieben". ${ }^{43}$ Zur zweiten Kölner Aufführung von Spohrs Jessonda am 9. Mai heißt es: „Ueber die Leistung des Orchesters bei dieser schwierigen Musik schweigen wir lieber. ${ }^{\text {" }} 4$ Zur Eröffnung des Aachener Theaters mit der Fessonda am 15. Mai 1825 rügte der dortige Rezensent Johann Baptist Rousseau (gleichzeitig Herausgeber der Zeitschrift Rheinische Flora, in der alle diese Kritiken erschienen), dass etliche Tempi, obwohl per Metronomangaben genau bezeichnet, völlig „vergriffen“ gewesen seien, und bemerkte: „es möge dem Herrn Musikdirektor gefallen, seine Zurufungen an's Orchester nicht so gar laut und die Illusion störend anzubringen [...]". ${ }^{45}$ Auch bei Mozarts Entführung aus dem Serail am 10. Juni wurde der Dirigierstil des Musikdirektors heftig angegriffen: „Das Orchester war brav, nur ging die Trommel zu dumpf. Herr v. Weber, der Gläserzerschmetterer, kann Forte und Presto angeben, ohne deswegen des Fußstampfens zu bedürfen. ${ }^{*}{ }^{46}$

Waren die Kritiker hingegen mit der Orchesterleistung zufrieden, wurden die ansässigen Musiker zwar gelobt, der „zugereiste“ Musikdirektor aber mit keiner Silbe erwähnt. ${ }^{47}$ Allerdings deuten Berichte auswärtiger Besucher darauf hin, dass es um die Qualität des Kölner Orchesters nicht zum Besten stand, so dass Edmund von Weber möglicherweise aufgrund des ihm zur Verfügung stehenden Personals resignierte. Der Londoner Sir George Smart sprach nach seinem Besuch einer Aufführung von Méhuls foseph am 16. November 1825 von einem „bad orchestra consisting of eight violins, two viole, two 'celli and two Bassi, with the usual number of wind instruments" ${ }_{48}$ und Wilhelm Speyer, der am 9. Mai 1825 eine Aufführung der fessonda besucht hatte, berichtete am 12. Mai dem befreundeten Komponisten Louis Spohr, das Orchester bestünde „fast gänzlich aus Greise[n], lauter 60 bis 70jährige Menschen“. ${ }^{49}$ Das Aache-

43 Rheinische Flora (wie Anm. 9), Jg. 1, Beilage zu Nr. 22 (6. Februar 1825).

44 Ebd., Jg. 1, Beilage zu Nr. 77 (15. Mai 1825).

45 Ebd., Jg. 1, Beilage zu Nr. 78 (17. Mai 1825).

46 Ebd., Jg. 1, Beilage zu Nr. 93 (14. Juni 1825).

47 Ebd., Jg. 1, Nr. 71 (5. Mai 1825), S. 284 (zu Fioravantis Sängerinnen auf dem Lande am 18. April in Köln: „Die Aufführung war sehr gelungen, und Ref. möchte behaupten, daß während des ganzen Winters keine Oper so präzise dargestellt wurde, als diese, sowohl von Seiten der Sänger und Sängerinnen, als des Orchesters“.), Nr. 79 (19. Mai 1825), S. 316 (zu Aubers Schnee am 17. Mai in Aachen: „Sänger und Orchester waren im besten Einklang“), Nr. 86 (2. Juni 1825), S. 344 (zu Mozarts Titus am 30. Mai in Aachen: „Das Orchester that alles nur Mögliche; die erste Klarinette, an der Stelle des Bassethorns, war unübertrefflich“.).

48 Hugh Bertram Cox und C. L. E. Cox (Hg.), Leaves from the fournals of Sir George Smart, London 1907, S. 221; demnach dirigierte Edmund von Weber selbst vom Geigenpult aus mit dem Bogen.

49 Vgl. Till Gerrit Waidelich, Die Beziehungen zwischen Carl Maria von Weber und Louis Spohr im Spiegel ihrer Korrespondenz, in: Weberiana. Mitteilungen der Internationalen Carl-Maria-von-Weber-Gesellschaft e. V. 24 (2014), S. 117-144, hier S. 138; Speyer kritisierte u. a. die Tempi. 
ner Orchester scheint zu jener Zeit das bessere gewesen zu sein. ${ }^{50}$ Die Angriffe auf Edmund von Weber in der örtlichen Presse nahmen mehr und mehr hämischen Charakter an: Zur Aufführung von Boieldieus Der neue Gutsherr am 4. Juli wurde glossiert: „im Orchester war Alles munter, nur Einer - schlief“" ${ }^{51}$ Dass damit der Musikdirektor gemeint war, bezeugt der Hinweis, dass Edmund von Weber im Don Giovanni am 20. Juli von einem solchen „magnetischen Schlaf überfallen“ wurde, dass er „zweimal den Ton nicht angab“ und dadurch „Sänger und Orchester gleich stark“ verwirrte: „Er mußte ordentlich von beiden Theilen fortgeschleppt werden. ${ }^{\text {"52 }}$ Auch anlässlich der Aufführung von Cherubinis Wasserträger am 3. August berichtete der Aachener Kritiker von „der apathiegleichen Art, wie jetzt bei uns die Oper dirigirt wird““ ${ }^{53}$ Selbst in der „Historische[n] Übersicht des Theaters in Aachen von 1825 bis Ende 1828“ in dem von Johann Sigismund Arendt herausgegebenen Theater-Almanach von 1829 liest man, dass das Aachener Orchester „von dem der [Ringelhardt'schen] Gesellschaft attachirten Musikdirektor, H e r r n v o n W e b e r (älterem Bruder des berühmten Komponisten) träge und schläfrig geleitet" wurde..$^{54}$ Nach der Rückkehr von Ringelhardts Truppe nach Köln urteilte der dortige Rezensent über die Preciosa-Darbietung am 28. Oktober: „Das Tempo der Ouvertüre sowie einiger anderer Musikstücke war zu langsam. ${ }^{\text {"5 }} \mathrm{Zu}$ Spontinis Vestalin am 30. November legte er noch nach:

Dann bleibt uns noch zu bemerken übrig, es möge doch dem Hrn. von Weber gefallen, die Partitur etwas genauer zu studiren: die Ouvertüre fängt mit einem Andante an und nicht mit einem Adagio, dann folgt ein Presto und kein Allegro. Ein Glück ist es, sowohl für das Publikum als die Sänger, daß diese gewöhnlich das Tempo selbst einsetzen, wo denn der Herr Taktschläger blos nach Anderer Gefühl den Takt sichtbar und hörbar nachschlägt. ${ }^{56}$

Anlässlich der Aufführung von Paers Sargines am 6. Januar 1826 in Aachen liest man die

Bemerkung, welche sowohl für diese als fast alle anderen Opern gilt, daß die Orchesterbegleitung bei uns meistens so stark zu sein pflegt,

50 Vgl. Rheinische Flora (wie Anm. 9), Jg. 1, Nr. 94 (16. Juni 1825), S. 378 (zu Fioravantis Sängerinnen auf dem Lande am 13. Juni): „Unsere Musiker bewährten ihren alten Ruhm, eines der besten Stadtorchester in Nähe und Ferne zu bilden."

51 Ebd., Jg. 1, Nr. 106 (7. Juli 1825), S. 426.

52 Ebd., Jg. 1, Nr. 115 (24. [recte 23.] Juli 1825), S. 462.

53 Ebd., Jg. 1, Nr. 123 (6. August 1825), S. 494.

54 Vgl. Almanach für's Aachener Stadt-Theater auf das fahr 1829, hg. von Dr. Arendt, Aachen 1829, S. 54; vgl. ebd., S. 75: „das Orchester kränkelte an schläfriger Leitung des Herrn von Weber“.

55 Rheinische Flora (wie Anm. 9), Jg. 1, Nr. 173 (3. November 1825), S. 692.

56 Ebd., Jg. 1, Beilage zu Nr. 193 (8. Dezember 1825). 
daß sie die Singstimme entweder ganz überflügelt oder zum mindesten benachtheiligt. Ein Pianissimo kommt höchst selten klar hervor, und bei dem Forte hört man nichts als das Stürmen der Instrumente; daß die Sängerin wirklich singt, kann man oft blos den Bewegungen ihres Mundes absehen. ${ }^{57}$

Und selbst Edmund von Webers Benefiz-Aufführung am 3. März 1826 in Köln gab Anlass zur Frage, was sich der Musikdirektor dabei gedacht habe, ausgerechnet „eine solche und so alte Oper" wie Grétrys Richard Löwenherz auszuwählen. ${ }^{58}$ Besonders in Aachen hielt der Spott an; zu Spontinis Vestalin am 14. Juli heißt es: „Der Musikdirektor war heute so fest an sein Pult, wie Prometheus an den Felsen, gebannt, daß er nicht im Stande war, der Virtuosität der Sänger und Sängerinnen zu folgen; er hinkte daher ihrem Auffluge nach. ${ }^{59}$ Immerhin konnte Edmund von Weber vor seinem Abschied von Aachen am 30. August 1826 in Spontinis Cortez (zu seinem Benefiz) nochmals sein Können unter Beweis stellen, so dass auch der Kritiker Rousseau die „sehr gelungene Aufführung“ anerkannte. ${ }^{60}$

In Detmold hatte sich Edmund von Weber aus dem öffentlichen Musikleben zunächst offenbar weitgehend zurückgezogen; Tochter Therese war die Hauptverdienerin der Familie. Nachdem die erhoffte Anstellung als Lehrer nicht zustande kam, dürfte er allerdings Orchesterdienste versehen haben. In der zweiten Jahreshälfte 1828 sprang er offenbar zunächst vertretungsweise ein, da er keine reguläre Gage erhielt, sondern lediglich innerhalb der Extra-Ausgaben für das Orchester aktenkundig wurde. ${ }^{61}$ Erst für die Monate Januar bis August 1829 liegen Gagenabrechnungen als festangestellter Orchestermusiker vor, nach welchen der „Alt-Violinist“ Edmund von Weber insgesamt 40 Taler erhielt; seine Wochengage (2 Taler, 12 Groschen) betrug weniger als ein Fünftel jener der Tochter Therese. ${ }^{62}$ Durch die Vermittlung der im Brief erwähnten Instrumente für die sogenannte ,Türkische Musik', so sie denn überhaupt zustande kam, dürfte er kaum mitverdient haben, aber durch Zusatzdienste als Notenkopist konnte er das Familieneinkommen aufbessern: Laut Rechnungen des Detmolder Hoftheaters

57 Rheinische Flora (wie Anm. 9), Jg. 2, Beilage zu Nr. 8 (10. Januar 1826).

58 Ebd., Jg. 2, Nr. 40 (11. März 1826), S. 160.

59 Ebd., Jg. 2, Beilage zu Nr. 111 (16. Juli 1826).

60 Ebd., Jg. 2, Beilage zu Nr. 138 (3. September 1826). Die Kölner Vorstellungen ab Herbst 1826 leitete bereits der neue Ringelhardt'sche Musikdirektor C. G. Woywoda; vgl. ebd., Jg. 2, Beilage zu Nr. 160 (12. Oktober 1826).

61 -DT, Theaterakten, TA 3 Haupt-Ausgabe zur Theater-Kasse 1828 und 1829, Tit. III Orchester 1828, S. 40 (August bis November): Nr. 35 (12 Taler, 12 Groschen), Nr. 37 (2 Taler), Nr. 52 (3 Taler, 8 Groschen „für Quartet-Proben“) und Nr. 54 (11 Taler, 16 Groschen).

62 Ebd., TA 3 Haupt-Ausgabe zur Theater-Kasse 1828 und 1829, Tit. I Gagen 1829, S. 94 (Nr. 35); außerdem das Gagenbuch Nr. 35 Edmund von Weber (Sign.: TA 101, 1829, Nr. 35). 
wurde er zwischen Januar und Mai 1829 für Teile der Aufführungsmaterialien zu Méhuls fe toller je besser, ${ }^{63}$ Dalayracs Die zwei Worte ${ }^{64}$ und Louis Maurers Aloise ${ }^{65}$ bezahlt.

Vermutlich Anfang September 1829 verließen die Webers Detmold. ${ }^{66}$ Vater Edmund dürfte bis zu seinem Tod seine Tochter Therese in ihre nachfolgenden Engagements begleitet haben. 1829 schloss sie zunächst einen Jahresvertrag am Hof- und Nationaltheater Mannheim ab (dortiges Debüt am 17. September 1829); ${ }^{67}$ von dort wechselte sie im Herbst 1830 erneut für ein Jahr nach Würzburg, wo die jüngere Schwester zudem eine Anstellung als Choristin bekam. ${ }^{68}$ In Würzburg starben bald nacheinander sowohl Edmund als auch seine Frau Therese. Für Edmund von Weber ist im Genealogischen Taschenbuch der Ritter- und Adels-Geschlechter das Todesjahr 1831 angegeben, ${ }^{69}$ allerdings fälschlich, denn das Intelligenz-Blatt für den Unter-Mainkreis des Königreichs Bayern nennt ihn bereits unter den im Dezember 1830 Verstorbenen. ${ }^{70}$ Für seine Frau

63 D-DT, Theaterakten, TA 3 Haupt-Ausgabe zur Theater-Kasse 1828 und 1829, Tit. IV für Musikalien, Januar 1829, Nr. 2 (3 Taler, 6 Groschen, 4 Pfennig).

64 Ebd., TA 3 Haupt-Ausgabe zur Theater-Kasse 1828 und 1829, Tit. IV für Musikalien, April 1829, Nr. 12 (7 Taler, 21 Groschen).

65 Ebd., TA 3 Haupt-Ausgabe zur Theater-Kasse 1828 und 1829, Tit. IV für Musikalien, Mai 1829, Nr. 27 (24 Taler, 15 Groschen); außerdem liegt ebd. eine nicht spezifizierte Abrechung für Kopialien vom Februar 1829 vor, Nr. 6 (2 Taler, 11 Groschen).

66 Die letzte Gage wurde am 30. August 1829 an Edmund von Weber gezahlt; vgl. das in Anm. 62 genannte Gagenbuch Nr. 35 für Edmund von Weber. Im September erhielten „Herr und Dem: Weber“ ein Reisegeld von 22 Talern, TA 3 Haupt-Ausgabe zur Theater-Kasse 1828 und 1829, Tit. II Diverse Ausgabe 1829, S. 107 (Nr. 219).

67 Vgl. Anton Pichler, Chronik des Großherzoglichen Hof- und National-Theaters Mannheim. Zur Feier seines hundertjährigen Bestehens am 7. October 1879, Mannheim 1879, S. 237 sowie Oscar Fambach, Das Repertorium des Hof- und Nationaltheaters in Mannheim 1804-1832, Bonn 1980 (Mitteilungen zur Theatergeschichte der Goethezeit 1), S. 540.

68 Vgl. Johann Georg Wenzel Dennerlein, Geschichte des Würzburger Theaters von seiner Entstehung im fahre 1803-4 bis zum 31. Mai 1853, nebst einem chronologischen Tagebuch und einem Anhang, Würzburg 1853, S. 232, 236. Auch Albert Lortzing erfuhr von der neuen Anstellung; vgl. den Brief an seine Eltern vom 7. November 1830 in Lortzing, Briefe (wie Anm. 7), S. 30.

69 Jg. 3, Brünn 1878, S. 784 f. Die dortigen Angaben zur Familie dürften auf Informationen von Edmunds Enkel Friedrich Hubert von Weber basieren.

70 Jg. 1831, Nr. 9 (25. Januar), Sp. 177 (in der „Bevölkerungs-Anzeige vom Monate December 1830“, präsentiert am 18. Januar 1831, unter den Gestorbenen: „Edmund von Weber, Musik-Direktor, 69 [recte 64] J. alt."). Clemens M. Gruber, Opern-Uraufführungen, Bd. 1, Wien 1994, S. 160, nennt als Sterbedatum genauer den 13. Dezember 1830. Die in der Literatur häufiger zu findende Behauptung, Edmund von Weber wäre nochmals bei Ringelhardt in dessen letzter Kölner Wintersaison 1831/32 engagiert gewesen, beruht auf einer Verwechslung mit einem seiner Söhne; vgl. Heyden, Kölner Theaterwesen (wie Anm. 3), S. 104 sowie Heinz Oepen, Beiträge zur Geschichte des Kölner Musiklebens 1760-1840, Köln 1955 (Beiträge zur rheinischen Musikgeschichte 10), S. 142. Richtig liest man hingegen in der Zeitschrift Allgemeine Theater-Chronik. Wöchentliche Mittheilungen von sämmtlichen deutschen Theatern, Jg. 1, Nr. 7 (12. Januar 1832), S. 27 in dem Bericht (datiert 29. Dezember 1831) über Ringelhardts Kölner Winterspielzeit (ab 
ist der Juli 1831 als Sterbemonat gesichert. In einer Münchner Zeitung findet sich zu dieser Zeit eine kurze Todesanzeige: „Auswärtig gestorben. [...] Würzburg: [...] Fr. Therese von Weber. Musikdirectorswittwe, 45 Jahre alt. ${ }^{\text {"71 }}$

Tochter Therese (ab 1833 verh. Atmer), die noch bis 1838 auf der Bühne aktiv war, ${ }^{72}$ lebte später in Berlin und lernte dort 1874, vermittelt durch ihren Cousin Max Maria von Weber, den Musikdirektor Friedrich Wilhelm Jähns kennen. ${ }^{73}$ Für Jähns zeichnete sie im Jahr darauf einige Erinnerungen an die Familie auf, die in der Jähns'schen Weberiana-Sammlung erhalten blieben..$^{74}$ Die aus großer zeitlicher Distanz niedergeschriebenen und somit leider in zahlreichen Details fehlerhaften Notizen gehen auch kurz auf die letzten fünf gemeinsam mit den Eltern verbrachten Jahre in Köln und Würzburg (ohne Erwähnung von Aachen, Detmold und Mannheim) ein:

In Cöln gefiel es uns Allen sehr gut; doch das Schicksal wurde nicht müde uns zu verfolgen. Meine Mutter begann zu kränkeln; mein Vater verspürte oft Brustschmerzen und heftigen Husten und als nun die furchtbare Nachricht von C. Marias Tode [am 5. Juni 1826] die Welt erschütterte, befiel meinen Vater eine schwere Krankheit. Als er sich scheinbar erholt, rieth ihm der Artzt nach Baden-Baden zu gehen..$^{75}$ Dort wurden ihm die theuren Briefe seines unvergesslichen Bruders gestohlen, die er in einem Kästchen mit sich führte und von welchem er sich nie trennte. So kam Schlag auf Schlag und der arme alte Mann, konnte sich nicht mehr erholen. Er kam wieder zurück, kränkelte noch einige Jahre und verschied dann an der Brustwassersucht, nachdem wir 1 Jahr früher nach Würzburg gezogen waren, theils der gesunden Luft wegen und theils weil Mutter dort einen geschickten Doktor gefunden zu haben glaubte, der ihre schrecklichen Leiden zu heilen hoffte. Diese Hoffnung war jedoch vergebens und nach $1 \frac{1}{2}$ Jahre[n] starb auch sie, uns beide mit 15 und 16 Jahren $[\mathrm{sic}]^{76}$ als Weisen zurücklassend. ${ }^{77}$

28. September 1831): „Der Musikdirektor H r . v. W e b e r, Neffe des berühmten Componisten, ist ein tüchtiger Mann, mit den gründlichsten Kenntnissen seines Faches ausgerüstet; er dirigirt mit Umsicht und Ruhe, und wir sehen unser Orchester unter seiner Leitung erfreulich gedeihen."

Der Bayerische Landbote, Jg. 7, Nr. 197 (25. Juli 1831), S. 978.

$72 \mathrm{Zu}$ ihrem späteren Lebensweg vgl. Frank Ziegler, Die Webers in Lauchstädt - Streiflichter zur Familienund regionalen Theatergeschichte, in: Weberiana. Mitteilungen der Internationalen Carl-Maria-von-WeberGesellschaft e. V. 15 (2005), S. 21-47, hier S. 43-46.

73 Vgl. den Brief von Max Maria von Weber an Jähns vom 28. Mai 1874 (Ostholstein-Museum Eutin, KHM Eutin Weber 63); mit dem Brief übersandte Weber Geld zur Unterstützung der offenbar bedürftigen Therese Atmer.

74 D-B, Weberiana Cl. V [Mappe XVIII], Abt. 4C, Nr. 44a.

75 Zur genaueren Datierung dieses Kuraufenthalts fehlen bislang dokumentarische Belege.

76 Die Schwestern Therese und Josephine waren beim Tod der Mutter fast 22 bzw. 20 Jahre alt.

77 D-B, Weberiana (wie Anm. 74). 
Wieviel tatsächliche Fakten dieser Text überliefert und wieviel phantasievolle Anreicherung, bleibt unklar. Die erwähnte Kränklichkeit des Vaters kann zumindest dessen vielgeschmähte Passivität in den letzten Dienstjahren als Musikdirektor erklären. Die Länge des Würzburg-Aufenthalts (angeblich ein Jahr bis zum Tod des Vaters Edmund und weitere anderthalb Jahre bis zum Tod der Mutter Therese) wurde auf jeden Fall übertrieben: Die Familie verbrachte dort weniger als ein Jahr. ${ }^{78}$

Der Lebensweg Edmund von Webers konnte in den letzten Jahren durch zahlreiche Quellenfunde im Rahmen der Recherchen zur Carl-Maria-von-Weber-Gesamtausgabe weitgehend rekonstruiert werden; trotzdem sind noch manche Details vage. Es bleibt zu hoffen, dass kommende Forschungen auch hierzu Klärung bringen!

78 Die Schwestern von Weber waren wohl kaum länger als ein halbes Jahr in Würzburg, da sie bei Dennerlein, Geschichte des Würzburger Theaters (wie Anm. 68), S. 232 erst für die Wintersaison (ab Oktober 1830) genannt werden. Therese von Weber befand sich im Februar 1831, also vor dem Tod der Mutter in Würzburg, bereits in Dessau; vgl. Ziegler, Die Webers in Lauchstädt (wie Anm. 72), S. 43 (Anm. 88) sowie Friedrich Dahm und August Gräge, Theater-fournal der vom 2. Februar bis 7. April 1831 unter der Direction des Herrn Heinr. Bethmann hieselbst gegebenen Vorstellungen, Dessau 1831. 